\title{
Fatores relacionado ao estresse em profissionais de saúde no combate à pandemia da COVID-19
}

Factors related to stress in health professionals in the fight against the COVID-19 pandemic Factores relacionados con el estrés en profesionales de la salud en la lucha contra la pandemia COVID-19

Paulo Sérgio da Paz Silva Filho ORCID: https://orcid.org/0000-0003-4104-6550 Centro Universitário Maurício de Nassau, Brasil E-mail: pauloosergio1@outlook.com

Alaíde Silva Lemos ORCID: https://orcid.org/0000-0003-4121-6724

Centro Universitário Uninovafapi, Brasil E-mail: laid_mel13@hotmail.com

Francisco Wagner dos Santos Sousa ORCID: https://orcid.org/0000-0001-9309-2925

Universidade Estadual do Piauí, Brasil E-mail:wagnersantosreal@gmail.com

Rosiane Leal dos Santos ORCID: https://orcid.org/0000-0002-0967-2642 Faculdade Metropolitana da Amazônia, Brasil E-mail: rose_leal_@hotmail.com

Francielly de Sousa Rodrigues ORCID https://orcid.org/0000-0002-5770-360X Universidade Federal do Piauí, Brasil E-mail: francyepr@hotmail.com

Alexandra Rodrigues Cardoso ORCID: https://orcid.org/0000-0002-7277-4668 Centro Universitário Maurício de Nassau, Brasil E-mail: alexandrarodcar@gmail.com Fernanda Maria Silva

ORCID: https://orcid.org/0000-0002-0504-9896 Universidade Regional do Cariri, Brasil E-mail: fernandamsmv@gmail.com

Ana Alinne Gomes Da Penha

ORCID: https://orcid.org/0000-0001-9253-1199 Universidade Regional do Cariri, Brasil E-mail: anaalinne.nurse@gmail.com Beatriz Silva Barros

ORCID: https://orcid.org/0000-0002-7470-1163 Centro Universitário Uninovafapi, Brasil E-mail: beatrizbarroos@ gmail.com

Fernanda da Silva Negreiros

ORCID: https://orcid.org/0000-0002-2326-4370 Centro universitário Uninovafapi, Brasil

E-mail: fernandasn-@hotmail.com

Maria Jandeline do Nascimento Silva ORCID: https://orcid.org/0000-0003-3337-3332 Centro Universitário INTA, Brasil

E-mail: jandelinesilvanascimento@gmail.com

Haroldo Bandeira de Matos Filho ORCID: https://orcid.org/0000-0003-0948-8017 ITPAC Porto nacional - TO, Brasil E-mail: haroldohrpn@hotmail.com Vinicius Pereira Colman ORCID: https://orcid.org/0000-0001-8547-9310 ITPAC Porto nacional - TO, Brasil

E-mail: viniciuspereiracolman@ hotmail.com Jardiel Marques Soares ORCID: https://orcid.org/0000-0003-0121-8223 Universidade Potiguar, Brasil 


\author{
E-mail: jardielmedicina@gmail.com \\ Tálison Vieira da Silva \\ ORCID: https://orcid.org/0000-0002-5628-8694 \\ Faculdade Ademar Rosado, Brasil \\ E-mail: profesptalison@gmail.com \\ Lillian Lettiere Bezerra Lemos Marques \\ ORCID: https://orcid.org/0000-0002-8261-8589 \\ Centro Universitário Maurício de Nassau, Brasil \\ E-mail: lillianlbl@gmail.com \\ Maria Regina Dos Santos Silva \\ ORCID: https://orcid.org/0000-0001-5721-1237 \\ Faculdade Pitágoras de São Luís, Brasil \\ E-mail: reginafarmsantos@gmail.com \\ Tayna Simone Mota da Silva \\ ORCID: https://orcid.org/0000-0002-6359-0242 \\ Faculdade Integrada Brasil Amazonia, Brasil \\ E-mail: tayna.simone@hotmail.com \\ Adayane Vieira Silva \\ ORCID: https://orcid.org/0000-0002-3802-6471 \\ Centro Universitário Maurício de Nassau, Brasil \\ E-mail: adayanne26@hotmail.com
}

\title{
Resumo
}

Trata-se de uma revisão integrativa da literatura, modalidade que consiste em resumir e analisar resultados de pesquisas. Para responder ao objetivo proposto foram consultadas as seguintes bases de dados: Web of Science (Clarivate Analytics), Google acadêmico, Medical Publications (PubMed), Scopus (Elsevier) e Scientific Electronic Library Online (SciELO). Foram utilizados os seguintes termos nas bases de dados: profissional de saúde, pandemia, COVID-19, coronavírus e saúde mental, dentro do recorte temporal de 2019 a 2021 . A amostra final do estudo foi de quinze artigos que atenderam ao objeto do estudo proposto. Na literatura analisada foram contempladas diferentes abordagens voltadas à minimização do estresse no ambiente laboral, diante da complexidade do fenômeno investigado. Dentre as estratégias capazes de amenizar o estresse em situação de pandemia estão as relacionadas ao ambiente e à jornada de trabalho, como a criação de sistemas capazes de gerenciar o estresse dos profissionais, flexibilização da jornada laboral e apoio destinado aos profissionais e seus familiares. O estudo é importante, pois a partir do conhecimento dos fatores que desencadeiam o estresse ocupacional destes profissionais, identifica estratégias que visam minimizar a ocorrência desse evento.

Palavras-chave: Profissional de saúde; Pandemia; COVID-19; Coronavírus; Saúde mental.

\begin{abstract}
This is an integrative literature review, which consists of summarizing and analyzing research results. To respond to the proposed objective, the following databases were consulted: Web of Science (Clarivate Analytics), Google academic, Medical Publications (PubMed), Scopus (Elsevier) and Scientific Electronic Library Online (SciELO). The following terms were used in the databases: health professional, pandemic, COVID-19, coronavirus and mental health, within the time frame from 2019 to 2021. The final study sample consisted of fifteen articles that met the proposed study object. In the analyzed literature, different approaches aimed at minimizing stress in the work environment were considered, given the complexity of the phenomenon investigated. Among the strategies capable of alleviating stress in a pandemic situation are those related to the environment and working hours, such as the creation of systems capable of managing the stress of professionals, flexibilization of working hours and support for professionals and their families. The study is important because, based on the knowledge of the factors that trigger the occupational stress of these professionals, it identifies strategies that aim to minimize the occurrence of this event.
\end{abstract}

Keywords: Health professional; Pandemic; COVID-19; Coronavirus; Mental health.

\section{Resumen}

Se trata de una revisión integrativa de la literatura, que consiste en resumir y analizar los resultados de la investigación. Para dar respuesta al objetivo propuesto se consultaron las siguientes bases de datos: Web of Science (Clarivate Analytics), Google academic, Medical Publications (PubMed), Scopus (Elsevier) y Scientific Electronic Library Online (SciELO). En las bases de datos se utilizaron los siguientes términos: profesional de la salud, pandemia, COVID-19, coronavirus y salud mental, en el marco temporal de 2019 a 2021. La muestra final del estudio estuvo conformada por quince artículos que cumplieron con el objeto de estudio propuesto. En la literatura analizada, se consideraron diferentes enfoques encaminados a minimizar el estrés en el ambiente de trabajo, dada la complejidad del fenómeno investigado. Entre las estrategias capaces de aliviar el estrés en situación de pandemia se encuentran las relacionadas con el medioambiente y el horario de trabajo, como la creación de sistemas capaces de gestionar el estrés de los profesionales, la flexibilización del horario laboral y el apoyo a los profesionales y sus familias. El estudio es importante porque, a partir del conocimiento de los factores que desencadenan el estrés laboral de estos profesionales, 
identifica estrategias que buscan minimizar la ocurrencia de este evento.

Palabras clave: Profesional de la salud; Pandemia; COVID-19; Coronavirus; Salud mental.

\section{Introdução}

A COVID-19 foi detectada pela primeira vez em dezembro de 2019 e, em março de 2020, foi declarada pandemia pela Organização Mundial da Saúde (OMS). O efeito rápido e agressivo do vírus causador da SARS-CoV-2 infectou milhões de humanos em 218 países e territórios e levou a mais de 1,4 milhão de mortes em todo o mundo em 24 de novembro de 2020.

Os coronavírus (CoVs) são um grande grupo de vírus conhecidos por causar infecções no trato respiratório superior em humanos, provocando quadros de resfriados comuns e infecções parecidos às da gripe. Seu nome se origina da presença de projeções de glicoproteínas em forma de taco (chamadas de pontas) que surgem da superfície do envelope viral e conferem uma aparência de coroa às partículas virais, semelhante à coroa do Sol, como visto na figura 1 (Mishra et al., 2021).

Figura 1 - Estrutura de uma partícula viral de um coronavírus.

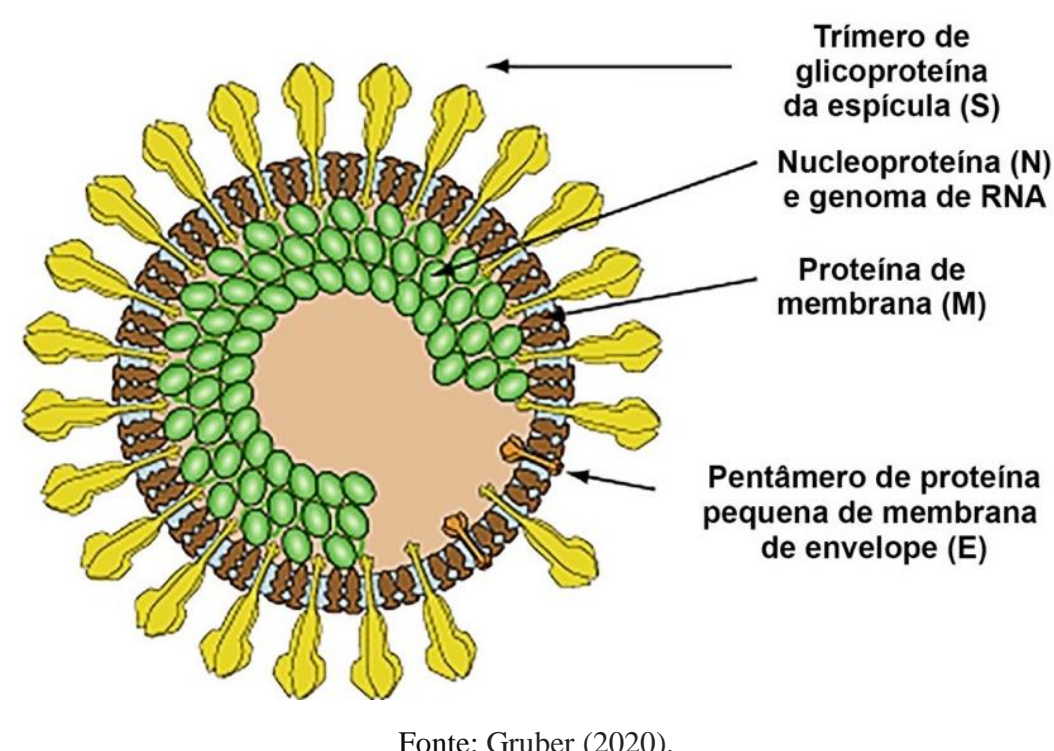

De acordo com o Protocolo de Manejo Clínico do Coronavírus, o vírus foi denominado coronavírus 2 da síndrome respiratória aguda grave (SARS-CoV-2) e produz a doença classificada como COVID-19. Os sintomas em sua maioria são febre, tosse seca, cansaço, coriza, obstrução nasal, dor de garganta e diarreia, porém já sabe-se que $14 \%$ dos pacientes apresentam sintomas mais graves como a dificuldade em respirar e falta de ar, havendo assim a necessitando de internação para oxigenoterapia e 5\% apresentam sintomas críticos (insuficiência respiratória, risco de morte, entre outros) (Prado et al., 2020).

Barroso et al., (2020) fizeram um mapeamento onde indicava o risco que os trabalhadores brasileiros têm de serem contaminados pelo COVID-19 durante suas atividades profissionais, a partir disso observou-se que os trabalhadores da saúde apresentaram de 97 a $100 \%$ de risco de contágio.

Contudo, a saúde física da população e o combate ao agente patogênico fazem parte do principal foco de gestores e profissionais da saúde, de modo que as implicações sobre a saúde mental tendem a ser negligenciadas ou subestimadas. Porém, é de extrema importância a realização de medidas para reduzir as implicações psicológicas da pandemia não podem ser desprezadas neste momento (Schmidt et al., 2020; Xiao, 2020). Com isso geram-se lacunas no combate dos desdobramentos negativos associados à doença, o que não é desejável (Ornell et al., 2020).

Borges et al., (2021), descreveram que a pandemia da COVID-19 ocasionou certa atenção com a saúde mental da 
sociedade, especialmente a dos profissionais de saúde que estão na linha de frente no combate a pandemia. Pois sabe-se que a jornada excessiva de trabalho provocado pelo novo coronavírus cresce de maneira desorganizada. Com isso, a sobrecarga de trabalho pode acarretar em problemas psicológicas e sociais, e interferir na qualidade de vida dos profissionais da saúde.

Os estudos sobre os impactos na saúde mental dos profissionais de saúde que lidam diretamente com as pessoas com populações infectadas pelo COVID-19 discutem a relação entre o temor pela exposição ao contágio, a situação de isolamento e confinamento e as medidas de quarentena implementadas. Entre os profissionais que estão ligados diariamente aos riscos de contaminação, em especial aqueles que trabalham em hospitais e postos de saúde, onde possível observar casos de exaustão, redução da empatia, ansiedade, irritabilidade, insônia e decaimento de funções cognitivas e do desempenho (Cruz et al., 2020).

Segundo Enumo et al. (2020) os casos mais comuns de estresse e ansiedade são de ordem: (a) física, como dor de cabeça, elevação dos batimentos cardíacos, alimentação desregularizada e de sono, úlceras, exaustão física; (b) emocionais, como tristeza, nervosismo, raiva, culpa, preocupação excessiva, perda de vontade e humor deprimido; (c) comportamentais, como irritabilidade, distanciamento, abuso de substâncias, violência; (d) cognitivos, como a perda de memória, dificuldade de concentração, dificuldade de tomar decisões. Com isso o presente estudo teve como objetivo descrever os principais fatores relacionado ao estresse em profissionais de saúde no combate à pandemia da COVID-19.

\section{Metodologia}

Trata-se de uma revisão integrativa da literatura, modalidade que consiste em resumir e analisar resultados de pesquisas, além divulgar sínteses de conhecimentos científicos produzidos acerca de um fenômeno de interesse (Siqueira; Santos \& Leonidas, 2020). A opção por essa modalidade de revisão se justifica por permitir a inclusão simultânea de diferentes tipos de estudos, cuja síntese oferece uma visão panorâmica do fenômeno de interesse.

A elaboração desta revisão integrativa seguiu sete etapas, como visto no Quadro 1.

Quadro 1 - Etapas da elaboração da revisão

\begin{tabular}{|c|c|}
\hline Etapas & \\
\hline $\mathbf{1}$ & Formação de um grupo para o desenvolvimento da revisão \\
\hline $\mathbf{2}$ & Elaboração da introdução \\
\hline $\mathbf{3}$ & Seleção do tema, formulação da pergunta e do objetivo \\
\hline $\mathbf{4}$ & Seleção dos artigos nas bases, análise crítica e interpretação dos estudos revisados \\
\hline $\mathbf{5}$ & Interpretação e discussão dos resultados \\
\hline $\mathbf{6}$ & Divulgação da revisão \\
\hline $\mathbf{7}$ & \\
\hline
\end{tabular}

\section{Estratégia de busca e questão norteadora}

Fonte: Whittemore \& Knafl (2005).

Para responder ao objetivo proposto foram consultadas as seguintes bases de dados: Web of Science (Clarivate Analytics), Google acadêmico, Medical Publications (PubMed), Scopus (Elsevier) e Scientific Electronic Library Online (SciELO). Na construção da estratégia de busca foram seguidos passos sistemáticos. Para cada base indexadora foi realizada uma adaptação dos termos/descritores utilizados na operacionalização da busca, dadas as características particulares de cada indexador. Essas fontes de indexação foram selecionadas por agruparem produções das áreas da saúde, da psicologia e estudos multidisciplinares. 
O estudo teve como questão norteadora indagar "Quais os principais fatores relacionado ao estresse associados aos profissionais de saúde no combate à pandemia da COVID-19?”. Para tanto, foi utilizado o modelo PVO, que contempla os seguintes elementos: P: situação problema, participantes e contexto (enfrentamento de pandemias, profissionais de saúde, cenário de conflagração mundial); V: variáveis dos estudos (indicadores de saúde física e mental); O: desfecho ou resultados (impactos ocupacionais). Esse modelo, por sua vez, foi adaptado da estratégia PICO - acrônimo que designa Paciente, Intervenção, Comparação e Outcomes -, usualmente utilizada em revisões sobre intervenção (Silva et al., 2014).

\section{Seleção dos estudos e extração de dados}

A operacionalização desta pesquisa iniciou-se com uma consulta ao conjunto de descritores consistentes com as bases escolhidas. Foram utilizados os seguintes termos nas bases de dados: profissional de saúde, pandemia, COVID-19, coronavírus e saúde mental. Os descritores foram utilizados de maneira combinada em português com o conector aditivo "e", e em buscas em inglês com o conector aditivo "and".

Definiram-se os seguintes critérios de inclusão dos estudos na revisão: artigos empíricos qualitativos e quantitativos; estudos teórico-reflexivos; dissertações, teses, livros, capítulos; estudos publicados em português, inglês e/ou espanhol, cujos resultados privilegiassem aspectos relacionados aos impactos relacionados ao estresse de profissionais de saúde durante a pandemia por covid-19. Dentro do recorte temporal de 2019 a 2021. Foram excluídos os editoriais, comentários e relatos de experiência. Também foram excluídos estudos que focalizam pacientes e suas vivências, além de artigos incompleto ou duplicados.

O processo de busca e seleção dos artigos foi realizado de forma independente pelos pesquisadores. Dúvidas ou inconsistências foram discutidas posteriormente, até que se estabelecessem os consensos. A busca foi operacionalizada no mês de novembro a dezembro de 2021. Em uma primeira fase foram avaliados os títulos e resumos dos artigos para, na sequência, ser realizada a leitura dos textos completos dos estudos selecionados.

\section{Análise dos dados}

Após a releitura de cada um dos artigos, os dados de interesse foram extraídos. Onde foi preenchido por meio de quadro com as seguintes informações: título, autores, periódico, ano de publicação, objetivos e conclusão. Os dados foram analisados de forma descritiva e independente pelos pesquisadores. Conforme preconizam as diretrizes para o desenvolvimento de revisões integrativas, foram sintetizados os principais resultados dos estudos, com foco nos dados que dialogavam com o objetivo da revisão. Todos os princípios éticos relacionados ao processo de construção de uma revisão integrativa de literatura foram observados, sendo que os estudos revisados e outros que foram incorporados ao manuscrito foram citados e referenciados.

\section{Resultados e Discussão}

A amostra final do estudo foi de quinze artigos que atenderam ao objeto do estudo proposto. Esse processo de inclusão, elegibilidade, seleção e identificação dos estudos foi disposto no Fluxograma 1. 
Fluxograma 1 - Registro dos estudos selecionados.

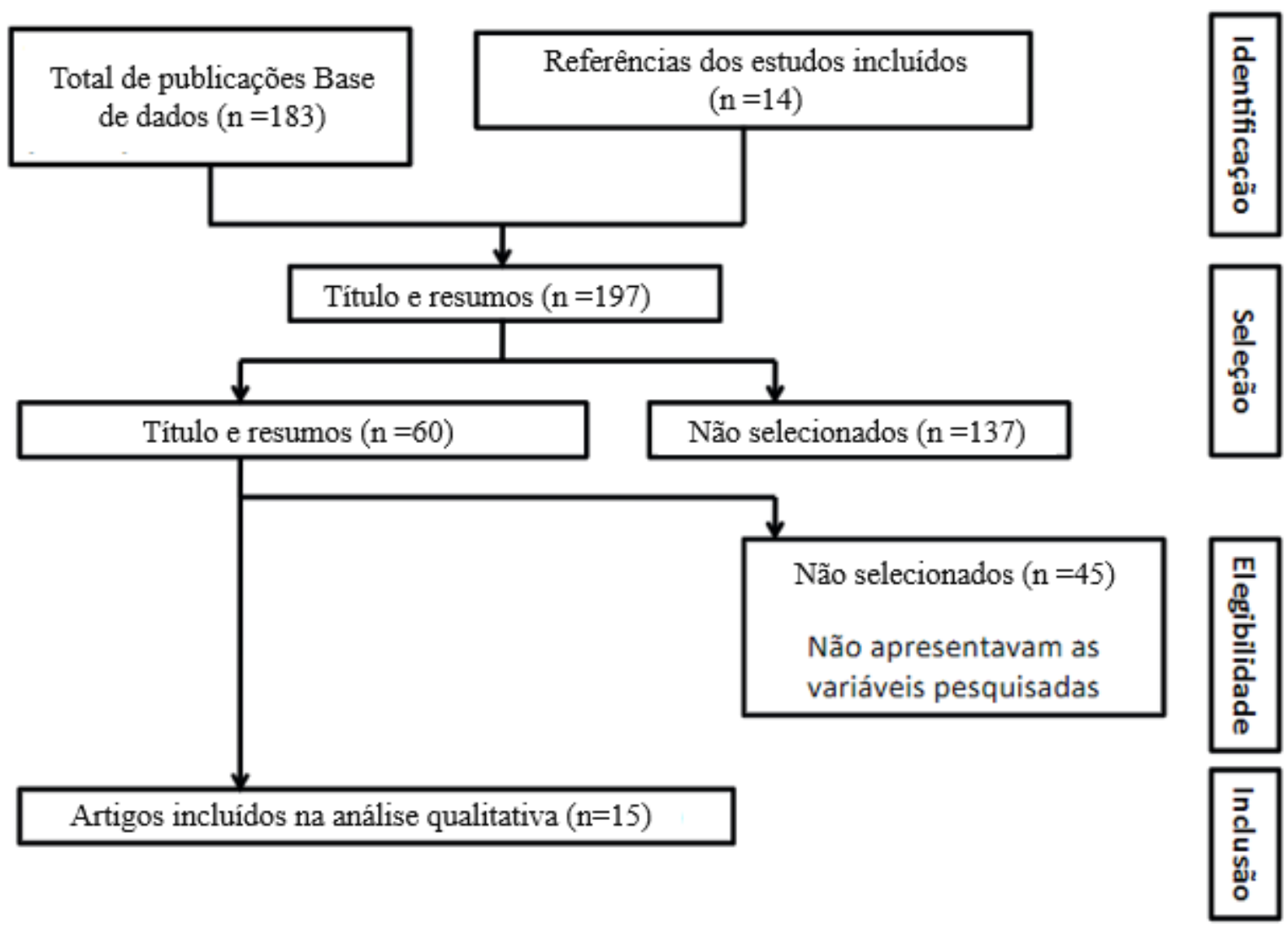

Fonte: Autores (2021).

Os artigos foram sintetizadas e caracterizadas em quadros sínteses para encontrar os anexos entre os artigos pesquisados, encontrando resposta à questão desta pesquisa, e objetivos propostos. Posteriormente, foi comparado ao referencial teórico que fundamentou o estudo. Para tal, foi realizado análise de conteúdo contendo Título do artigo, autores/ano, Objetivos e Conclusão, como pode ser observado no Quadro 2:

Quadro 2 - Caracterização dos artigos.

\begin{tabular}{|c|c|c|c|}
\hline TITULO & AUTORES / ANO & OBJETIVO & CONCLUSÃO \\
\hline $\begin{array}{c}\text { Pandemia do coronavírus: } \\
\text { estratégias amenizadoras do } \\
\text { estresse ocupacional em } \\
\text { trabalhadores da saúde. }\end{array}$ & $\begin{array}{c}\text { (Sousa Júnior et al., } \\
\text { 2020) }\end{array}$ & $\begin{array}{c}\text { Sistematizar conhecimentos sobre } \\
\text { as estratégias amenizadoras do } \\
\text { estresse ocupacional em } \\
\text { trabalhadores da saúde durante a } \\
\text { pandemia do coronavírus. }\end{array}$ & $\begin{array}{c}\text { As estratégias para enfrentamento e } \\
\text { controle dos agentes estressores em } \\
\text { profissionais de saúde incluem ações } \\
\text { para promover à saúde mental, } \\
\text { autoajuda e espiritualidade, programas } \\
\text { de gestão e ações educativas. }\end{array}$ \\
\hline $\begin{array}{c}\text { Fatores de estresse nos } \\
\text { profissionais de enfermagem } \\
\text { no combate à pandemia da } \\
\text { COVID-19: síntese de } \\
\text { evidências. }\end{array}$ & (Barbosa et al. 2020) & $\begin{array}{c}\text { Identificar os principais efeitos } \\
\text { psicológicos da pandemia da } \\
\text { COVID-19 nos profissionais de } \\
\text { enfermagem; descrever os } \\
\text { principais fatores capazes de } \\
\text { gerar estresse psicológico nos } \\
\text { professionais de enfermagem; } \\
\text { descrever as estratégias de coping } \\
\text { para o combate ao estresse } \\
\text { emocional. }\end{array}$ & $\begin{array}{c}\text { É de suma importância considerar as } \\
\text { questões psicológicas, reconhecendo e } \\
\text { acolhendo os receios e medos dos } \\
\text { profissionais de enfermagem criando-se } \\
\text { assim uma esfera de estabilidade em } \\
\text { meio à crise. }\end{array}$ \\
$\begin{array}{c}\text { The Impact of COVID-19 } \\
\text { Epidemic Declaration on } \\
\text { Psychological Consequences: }\end{array}$ & (Li et al., 2020). & $\begin{array}{c}\text { The aim of this study is to explore } \\
\text { the impacts of public health } \\
\text { emergency COVID-19 on }\end{array}$ & $\begin{array}{c}\text { We found an increase in negative } \\
\text { emotions (anxiety, depression, and } \\
\text { indignation) and sensitivity to social }\end{array}$ \\
\hline
\end{tabular}




\begin{tabular}{|c|c|c|c|}
\hline $\begin{array}{l}\text { A Study on Active Weibo } \\
\text { Users. }\end{array}$ & & people's mental health & $\begin{array}{l}\text { risks, as well as a decrease in positive } \\
\text { emotions (Oxford happiness) and life } \\
\text { satisfaction after declaration of } \\
\text { COVID-19 in China. }\end{array}$ \\
\hline $\begin{array}{l}\text { Psychiatric symptomatology } \\
\text { associated with depression, } \\
\text { anxiety, distress, and } \\
\text { insomnia in health } \\
\text { professionals working in } \\
\text { patients affected by } \\
\text { COVID19: a systematic } \\
\text { review with meta-analysis. }\end{array}$ & (Silva \& Neto, 2020). & $\begin{array}{l}\text { Describe the main psychiatric } \\
\text { symptoms associated with } \\
\text { depression, anxiety, anxiety and } \\
\text { insomnia in health professionals } \\
\text { working with patients affected by } \\
\text { COVID-19 }\end{array}$ & $\begin{array}{l}\text { There is a strong association between } \\
\text { health professionals and COVID-19 in } \\
\text { terms of psychiatric repercussions. }\end{array}$ \\
\hline $\begin{array}{l}\text { Adoecimento mental na } \\
\text { população geral e } \\
\text { profissionais de saúde } \\
\text { durante a pandemia da covid- } \\
\text { 19: revisão sistemática. }\end{array}$ & (Moreira et al. 2020) & $\begin{array}{l}\text { Identificar e analisar a literatura } \\
\text { sobre adoecimento mental na } \\
\text { população geral e profissionais de } \\
\text { saúde no contexto da pandemia } \\
\text { do novo coronavírus. }\end{array}$ & $\begin{array}{l}\text { A pandemia do novo coronavírus } \\
\text { desencadeou, com maior frequência, } \\
\text { quadros de ansiedade, depressão, } \\
\text { estresse e transtornos do estresse pós- } \\
\text { traumático na população geral e } \\
\text { profissionais de saúde. Mulheres, } \\
\text { estudantes e enfermeiros estão entre os } \\
\text { mais acometidos. }\end{array}$ \\
\hline $\begin{array}{l}\text { Dealing with psychological } \\
\text { distress by healthcare } \\
\text { professionals during the } \\
\text { COVID-19 pandemia. }\end{array}$ & (Petzold et al. 2020) & $\begin{array}{l}\text { This article summarizes the first } \\
\text { recommendations for the } \\
\text { reduction of stress and } \\
\text { psychological burden in health } \\
\text { professionals during the current } \\
\text { COVID-19 pandemia. }\end{array}$ & $\begin{array}{l}\text { This article presents guidelines for } \\
\text { health professionals and team leaders in } \\
\text { healthcare that help to maintain mental } \\
\text { health during the COVID-19 pandemia. }\end{array}$ \\
\hline $\begin{array}{l}\text { Coronavirus disease } 2019 \\
\text { (COVID-19) and beyond: } \\
\text { micropractices for burnout } \\
\text { prevention and emotional } \\
\text { wellness. }\end{array}$ & $\begin{array}{l}\text { (Fessell \& Cherniss, } \\
\text { 2020). }\end{array}$ & $\begin{array}{l}\text { Describe Micropractices for } \\
\text { burnout prevention and emotional } \\
\text { well-being }\end{array}$ & $\begin{array}{l}\text { As the demands and stresses of } \\
\text { COVID- } 19 \text { evolve, we can use and } \\
\text { build our resources-both internal and } \\
\text { external. Micropractice by } \\
\text { micropractice, breath by breath, we can } \\
\text { move toward increased calm and } \\
\text { elevated emotional wellness. }\end{array}$ \\
\hline $\begin{array}{l}\text { Estratégias nas instituições de } \\
\text { saúde para reduzir estresse na } \\
\text { enfermagem. }\end{array}$ & $\begin{array}{l}\text { (Calil \& Francisco, } \\
\text { 2020) }\end{array}$ & $\begin{array}{c}\text { Identificar as principais } \\
\text { estratégias utilizadas pelas } \\
\text { instituições de saúde para } \\
\text { diminuir o estresse entre a equipe } \\
\text { de enfermagem. }\end{array}$ & $\begin{array}{l}\text { Com os altos índices de estresse que } \\
\text { acometem as equipes de instituições de } \\
\text { saúde, a identificação de fatores } \\
\text { estressores no trabalho corresponde a } \\
\text { um dos grandes agentes de mudança, } \\
\text { facilitando o planejamento e a } \\
\text { implementação de ações para melhoria } \\
\text { da qualidade de vida e de trabalho dos } \\
\text { profissionais gestores dos serviços de } \\
\text { saúde. }\end{array}$ \\
\hline $\begin{array}{c}\text { Fatores de risco para a } \\
\text { Síndrome de Burnout em } \\
\text { profissionais da saúde } \\
\text { durante a pandemia de } \\
\text { Covid-19. }\end{array}$ & (Borges et al. 2021) & $\begin{array}{c}\text { Analisar os fatores de risco para o } \\
\text { desenvolvimento da Síndrome de } \\
\text { Burnout em profissionais da } \\
\text { saúde durante a pandemia da } \\
\text { COVID-19. }\end{array}$ & $\begin{array}{l}\text { Medidas de intervenção psicológica } \\
\text { devem ser implementadas para } \\
\text { minimizar os efeitos dos fatores que } \\
\text { contribuem para o desenvolvimento da } \\
\text { Síndrome de Burnout nos profissionais } \\
\text { de saúde. }\end{array}$ \\
\hline $\begin{array}{l}\text { Mental health outcomes of the } \\
\text { CoViD-19 pandemic. }\end{array}$ & (Talevi et. al., 2020). & $\begin{array}{l}\text { This review aims at synthesizing } \\
\text { mounting evidence concerning } \\
\text { the immediate psychological } \\
\text { responses during the initial stage } \\
\text { of the CoViD-19 pandemic } \\
\text { among the general population, the } \\
\text { health-care workers, and clinical } \\
\text { populations. }\end{array}$ & $\begin{array}{l}\text { A new model of psychological crisis } \\
\text { intervention is needed to better address } \\
\text { the pressing psychological problems of } \\
\text { people affected by the CoViD-19 } \\
\text { pandemic. Online mental health } \\
\text { services have been widely adopted in } \\
\text { China and are promoted in other } \\
\text { countries due to the recommendation to } \\
\text { minimize face-to-face interactions. }\end{array}$ \\
\hline $\begin{array}{l}\text { Meditação na redução do } \\
\text { estresse em profissionais de } \\
\text { saúde na pandemia do Covid- } \\
\text { 19: revisão narrativa. }\end{array}$ & (Melo et al. 2020), & $\begin{array}{l}\text { Descrever a influência da } \\
\text { meditação para atenuar o estresse } \\
\text { dos profissionais de saúde } \\
\text { expostos aos riscos de } \\
\text { adoecimento psico-lógico frente à } \\
\text { pandemia do COVID-19. }\end{array}$ & $\begin{array}{l}\text { A pandemia e as medidas de } \\
\text { enfrentamento ao COVID- } 19 \text { criam } \\
\text { fatores de estresse para os profissionais } \\
\text { de saúde pois estão em situação de } \\
\text { risco. O estresse representa um risco à } \\
\text { saúde fí-sica e mental, podendo } \\
\text { interferir diretamente no seu } \\
\text { crescimento e desenvolvimento pessoal } \\
\text { e profissional }\end{array}$ \\
\hline
\end{tabular}




\begin{tabular}{|c|c|c|c|}
\hline $\begin{array}{c}\text { Mental health of healthcare } \\
\text { professionals in China during } \\
\text { the new coronavirus } \\
\text { pandemic: an integrative } \\
\text { review. }\end{array}$ & (Paiano et al. 2020) & $\begin{array}{l}\text { to identify publishing related to } \\
\text { the mental health of health } \\
\text { professionals working in the front } \\
\text { line of the COVID-19 pandemic. }\end{array}$ & $\begin{array}{l}\text { All mentioned aspects have a direct } \\
\text { impact on the mental health of } \\
\text { professionals, demanding the creation } \\
\text { of strategies that minimize the } \\
\text { emotional burnout of workers, } \\
\text { considering that each country and } \\
\text { culture reacts differently to the disease. }\end{array}$ \\
\hline $\begin{array}{l}\text { The mental health of medical } \\
\text { workers in Wuhan, China } \\
\text { dealing with the } 2019 \text { novel } \\
\text { coronavirus. }\end{array}$ & (Kang et al., 2020). & $\begin{array}{l}\text { Relatar como anda a saúde mental } \\
\text { dos trabalhadores médicos em } \\
\text { Wuhan, China, lidando com o } \\
\text { novo coronavírus de } 2019\end{array}$ & $\begin{array}{l}\text { Esta publicação marca a primeira vez } \\
\text { que uma orientação para fornecer } \\
\text { proteção psicológica multifacetada da } \\
\text { saúde mental de trabalhadores médicos } \\
\text { foi iniciada na China. As experiências } \\
\text { desta emergência de saúde pública } \\
\text { devem informar a eficiência e a } \\
\text { qualidade da futura intervenção do } \\
\text { governo chinês e das autoridades em } \\
\text { todo o mundo em crises. }\end{array}$ \\
\hline $\begin{array}{l}\text { O impacto da pandemia por } \\
\text { COVID-19 na saúde mental } \\
\text { dos profissionais de saúde: } \\
\text { revisão integrativa. }\end{array}$ & (Bezerra et al. 2020) & $\begin{array}{l}\text { Identificar os fatores que } \\
\text { impactam na saúde mental dos } \\
\text { profissionais da saúde no } \\
\text { enfrentamento da COVID-19. }\end{array}$ & $\begin{array}{l}\text { Conclui-se que, os profissionais da } \\
\text { saúde que atuam na linha de frente em } \\
\text { meio à pandemia do coronavírus, mais } \\
\text { do que nunca, encontram-se em } \\
\text { situações estressantes, o que acarreta } \\
\text { uma sobrecarga maior de preocupação, } \\
\text { trabalho, ansiedade e depressão diante } \\
\text { de tantas mortes e longos turnos de } \\
\text { trabalho. }\end{array}$ \\
\hline $\begin{array}{l}\text { Psychological status of } \\
\text { medical workforce during the } \\
\text { COVID-19 pandemic: A } \\
\text { cross-sectional study. }\end{array}$ & (Lu et al., 2020) & $\begin{array}{l}\text { Describe Psychological status of } \\
\text { the medical workforce during the } \\
\text { COVID-19 pandemic }\end{array}$ & $\begin{array}{l}\text { The current study found support that the } \\
\text { medical staff unfolded greater fear, } \\
\text { anxiety and depression than the } \\
\text { administrative staff. Moreover, the front } \\
\text { line medical staff working in } \\
\text { department of } \\
\text { respiratory, emergency, ICU and } \\
\text { infectious disease, were } \\
\text { twice more likely to suffer anxiety and } \\
\text { depression than the non-clinical staff } \\
\text { with hardly possibility to contact with } \\
\text { coronavirus pneumonia patients. }\end{array}$ \\
\hline
\end{tabular}

Fonte: Autores (2021).

$\mathrm{Na}$ literatura analisada foram contempladas diferentes abordagens voltadas à minimização do estresse no ambiente laboral, diante da complexidade do fenômeno investigado. Dentre as estratégias capazes de amenizar o estresse em situação de pandemia estão as relacionadas ao ambiente e à jornada de trabalho, como a criação de sistemas capazes de gerenciar o estresse dos profissionais, flexibilização da jornada laboral e apoio destinado aos profissionais e seus familiares.

Os profissionais da saúde estão na linha de frente para resposta a pandemia ocasionada pela COVID-19 e, com isso, acabam ficando mais vulneráveis a infecção. Os perigos incluem maior exposição ao patógeno, longas jornadas de trabalho, estresse emocional, fadiga, síndrome do esgotamento físico e mental inerente ao trabalho (síndrome de Burnout), estigma e violência física e psicológica (Sousa Júnior et al., 2020).

Barbosa et al. (2020), relataram que os profissionais de saúde, é a categoria populacional mais acometida psicologicamente, sendo que passam por fatores estressores adicionais tais como: aumento da carga de trabalho, medo de contaminar os familiares e também de se contaminar, desinformação e raiva do governo e dos sistemas de saúde. Além disso, a elevada quantidade de doentes e mortes no ocasionado pela pandemia gera um alto risco psicossocial ocupacional, para as equipes que atuam na linha de frente.

O estresse é tido como um momento de tensão, onde ocorre a alteração da homeostase de uma pessoa, sendo percebido, inicialmente, por sinais e sintomas psicossomáticos, no geral é acompanhado por: taquicardia, gastrite, alterações 
cardiovasculares, insônia e outros. No ambiente de trabalho, esse fenômeno é responsável por afetar negativamente a qualidade de vida dos profissionais (Li et al., 2020; Sousa Júnior et al., 2020).

No estudo realizado por Moreira et al. (2020), descreveram que durante o estágio inicial da pandemia cerca de 1.210 indivíduos de 194 cidades da China, mostrou que 53,8\% classificaram o impacto psicológico da doença como moderado ou grave, onde $28,8 \%, 16,5 \%$ e $8,1 \%$ estavam relacionados a sintomas moderados ou graves de ansiedade, depressão e estresse, respectivamente.

Petzold et al. (2020), após relatarem os principais fatores capazes de causar estresse emocional apresentaram as diretrizes para profissionais de saúde e líderes de equipe de saúde na luta contra o estresse emocional atrelado a pandemia da COVID-19. Além disso, os pesquisadores buscaram associar a satisfação de necessidades básicas, o apoio social, a comunicação, a distribuição clara de tarefas, os horários flexíveis de trabalho e a utilização de ajuda psicossocial e psicológica sem estigmatização, onde são medidas particularmente mais importantes na redução do estresse emocional ao qual profissionais de saúde estão submetidos.

No estudo de Borges et al. (2021), foi realizada uma análise dos principais fatores de estresse ao profissional de saúde no combate a COVID-19 identificado nos artigos, como visto no Quadro 3.

Quadro 3 - Fatores de estresse ao profissional de saúde no combate à Covid-19.

\begin{tabular}{|c|}
\hline \multicolumn{1}{|c|}{ Fatores de Estresse } \\
\hline I- Aumento do número de casos confirmados esuspeitos. \\
\hline II- Contato próximo com pacientes infectados. \\
III- Distanciamento da família e amigos. \\
IV- Carga horária de trabalho exaustiva. \\
\hline V- Falta de Equipamentos de Proteção Individuais (EPIs). \\
\hline VI- Falta de medicamentos específicos. \\
VII- A cobertura da mídia. \\
\hline VIII- Sentimentos de apoio inadequados. \\
\hline IX- risco de contrair o vírus e a preocupação decontágio de seus familiares. \\
\hline X- O estresse e a pressão de lidar com o seu trabalho,acrescido do risco de adoecer. \\
XI- Risco de ser infectado, adoecer ou morrer, além \\
da possibilidade e medo de infectar outras pessoas. \\
\hline XII- Exposição a mortes em larga escala e afrustração pela perda da vida de seus \\
pacientes.
\end{tabular}

Fonte: Adaptado de Borges et al. (2021).

O estudo ainda aponta que a elevação de casos confirmados e suspeitos nos ambientes hospitalares. Pois desde o início da pandemia de coronavírus, os casos só aumentaram, tal confirmação gerou preocupação na população e necessidade de mais investimento em estrutura hospitalar para atender ao aumento da demanda pelos serviços de saúde, sendo esse um fator de estresse aos profissionais.

O fator II do quadro descreve a respeito do contato próximo com pacientes infectados, visto isso, sabe-se que os profissionais que atuam na linha de frente com os pacientes acometidos por COVID-19 descrevem uma maior gravidade dos sintomas quando comparado aos demais profissionais (Talevi et. al., 2020; Silva et al.,, 2020).

A jornada de trabalho também é um fator que gera estresse entre os profissionais da saúde, estudos descrevem que a 
sobrecarga de trabalho durante certo momento da pandemia pode levar ao esgotamento físico, onde pode acarretar de maneira negativamente a assistência ao paciente (Fessell et al., 2020). Já Calil et al., (2020), relatam que traz o excesso de trabalho como um fator relacionado ao cansaço e redutor do autocuidado e lazer e, pode levar ao aumento dos níveis de estresse. Portanto, é imprescindível que as instituições de saúde não proporcionem aos seus profissionais longas jornadas de trabalho, principalmente em momentos de pandemias.

Na pesquisa realizada por Melo et al. (2020), descreveram que no cenário brasileiro de assistência à saúde, existem diversos fatores relacionado ao estresse de profissionais de saúde, como alta carga de trabalho, falta de profissionais capacitados, a falta de dispositivos de Equipamento de Proteção Individual (EPI) e Equipamento de Proteção Coletiva (EPC), sistema de controle de infecção não eficaz e, surpreendentemente, ainda há o insulto direto dos pacientes aos profissionais que estão na linha de frente no combate ao COVID-19, como insulto verbal e físico.

Paiano et al. (2020) cita que um grande problema relacionado aos equipamentos de proteção individual á que devido sua precariedade, os profissionais necessitam conservá-los por um longo período, onde muitas vezes acarreta no desconforto e fadiga, uma vez que, normalmente, atrelado a isso observou-se que os profissionais não podem consumir alimentos ou ir ao banheiro por cerca de 6 horas afim de evitar a contaminação do equipamento.

Além do receio do próprio contágio, os servidores da saúde atam com receio da infecção à sua família, colegas de trabalho e demais pessoas próximo a ele, como visto no item IX do quadro 3, além disso foi possível identificar o sentindo incertezas e rotulações, relutâncias em ir trabalhar e altos índices de pedidos de demissão. Alguns desses profissionais estão trabalhando isoladamente, em alta exposição aos riscos e tem e/ou tiveram contato com colegas de trabalho que estão/foram contaminados, havendo também o relato de alguns casos de óbito, em um período de aproximadamente quatro meses (Kang et al., 2020).

O mesmo foi descrito no estudo de Bezerra et al. (2020), onde foi relatado como fatores relacionados à vínculos familiares e de amizade também foram associados à deficiência voltada a saúde mental da equipe de saúde, onde foi descrito em alguns casos o falecimento de colegas, hospitalizado ou em quarentena devido a COVID-19, onde isso acarretava ao aparecimento de transtorno de estresse pós-traumático.

É importante ressaltar o acompanhamento de equipes voltada a saúde mental do trabalhador, entre elas: psiquiatras, psicólogos, enfermeiros e outros profissionais de saúde para dar suporte no enfrentamento da tensão e reduzir o risco de ansiedade e depressão da equipe médica. Realizar organização abrangente de consultas psicológicas regular e por um longo tempo, para tratar aqueles profissionais que sofrerem de estresse pós-traumático (Lu et al., 2020).

\section{Considerações Finais}

O estudo é importante, pois a partir do conhecimento dos fatores que desencadeiam o estresse ocupacional destes profissionais, identifica estratégias que visam minimizar a ocorrência desse evento.

Após as evidências científicas demostrarem que a equipe de saúde tem passado por sofrimento psicológico com a pandemia do COVID-19, percebeu-se a importância de tratamentos psicológicos ou psiquiátricos à essa população, uma vez que, o cuidado em saúde mental favorece a atuação do profissional no seu local de trabalho, e a ausência disso reduzirá o seu potencial de cuidado, aumentarão as chances de afastamentos, disseminações, mortes e consequências posteriores a crise desta pandemia.

Enquanto as curvas dos indicadores de infecção pelo novo coronavírus diminuem mundialmente, as consequências relacionadas à saúde mental persistem e podem gerar efeitos danosos a longo prazo, fazendo-se urgente e necessárias ações estratégicas individuais e de abrangência comunitária que minimizem a ocorrência, deteriorações emocionais e agravos psicológicos na população e equipes de saúde, uma vez que estas não podem ser negligenciadas. 
As limitações do estudo pautam-se na utilização de poucos textos na amostra final, tendo em vista a contemporaneidade do tema e a existência de poucos artigos que enfoquem a temática saúde mental, estresse e profissionais da saúde.

\section{Referências}

Barbosa, D. J., Gomes, M. P., de Souza, F. B. A., \& Gomes, A. M. T. (2020). Fatores de estresse nos profissionais de enfermagem no combate à pandemia da COVID-19: síntese de evidências. Comunicação em Ciências da Saúde, 31, 31-47.

Barroso, B. I., de Souza, M. B. C. A., Bregalda, M. M., Lancman, S., \& da Costa, V. B. B. Saúde do trabalhador em tempos de covid-19: reflexões sobre saúde, segurança e terapia ocupacional.

Bezerra, G. D., Sena, A. S. R., Braga, S. T., dos Santos, M. E. N., Correia, L. F. R., de Freitas Clementino, K. M., ... \& Pinheiro, W. R. (2020). O impacto da pandemia por COVID-19 na saúde mental dos profissionais de saúde: revisão integrativa. Revista Enfermagem Atual In Derme, 93.

Borges, F. E. D. S., Aragão, D. F. B., Borges, F. E. S., Borges, F. E. S., SOUSA, A., \& Machado, A. L. G. (2021). Fatores de risco para a Síndrome de Burnout em profissionais da saúde durante a pandemia de Covid-19. Rev Enferm Atual In Derme, 95(33).

Calil, T. Z. N., \& Francisco, C. M. (2020). Estratégias nas instituições de saúde para reduzir estresse na enfermagem. Revista Recien-Revista Científica de Enfermagem, 10(29), 40-47.

Cruz, R. M., Borges-Andrade, J. E., Moscon, D. C. B., Micheletto, M. R. D., Esteves, G. G. L., Delben, P. B., ... \& Carlotto, P. A. C. (2020). COVID-19: emergência e impactos na saúde e no trabalho. Revista Psicologia Organizações e Trabalho, 20(2), I-III.

Enumo, S. R. F., Weide, J. N., Vicentini, E. C. C., Araujo, M. F. D., \& Machado, W. D. L. (2020). Enfrentando o estresse em tempos de pandemia: proposição de uma cartilha. Estudos de Psicologia (Campinas), 37.

Fessell, D., \& Cherniss, C. (2020). Coronavirus disease 2019 (COVID-19) and beyond: micropractices for burnout prevention and emotional wellness. Journal of the american college of radiology, $17(6), 746$

Gruber, A. (2020). Covid-19: o que se sabe sobre a origem da doença. Jornal da Universidade de São Paulo.

Kang, L., Li, Y., Hu, S., Chen, M., Yang, C., Yang, B. X., ... \& Liu, Z. (2020). The mental health of medical workers in Wuhan, China dealing with the 2019 novel coronavirus. The Lancet Psychiatry.

Li,et al. (2020). The Impact of COVID-19 Epidemic Declaration on Psychological Consequences: A Study on Active Weibo Users. International Journal of Environmental Research and Public Health, 2032(17),1-9.v

Lu, W., Wang, H., Lin, Y., \& Li, L. (2020). Psychological status of medical workforce during the COVID-19 pandemic: A cross-sectional study. Psychiatry research, 288, 112936 .

Melo, B. C., Souza, B. T. T., Pancheniak, G., Rauber, S. B., de Jesus, B., Guedes, M. R., ... \& de Freitas, P. H. D. (2020). Meditação na redução do estresse em profissionais de saúde na pandemia do Covid-19: revisão narrativa. Comunicação em Ciências da Saúde, 31(03), 65-70.

Mishra, SK e Tripathi, T. (2021). Atualização de um ano sobre a pandemia COVID-19: Onde estamos agora ?. Acta tropica , 214 , 105778.

Moreira, W. C., de Sousa, A. R., Maria do Perpétuo, S. S., \& Moreira, W. C. (2020). Adoecimento mental na população geral e profissionais de saúde durante a pandemia da covid-19: revisão sistemática. Texto \& Contexto Enfermagem, 29(1).

Ornell, F., Schuch, J. B., Sordi, A. O., \& Kessler, F. H. P. (2020). "Pandemic fear” and COVID-19: mental health burden and strategies. Brazilian Journal of Psychiatry, 42(3), 232-235.

Paiano, M., Jaques, A. E., Nacamura, P. A. B., Salci, M. A., Radovanovic, C. A. T., \& Carreira, L. (2020). Mental health of healthcare professionals in China during the new coronavirus pandemic: an integrative review. Revista Brasileira de Enfermagem, 73.

Petzold, M. B., Plag, J., \& Ströhle, A. (2020). Dealing with psychological distress by healthcare professionals during the COVID-19 pandemia. Der Nervenarzt

Prado, A. D., Peixoto, B. C., da Silva, A. M. B., \& Scalia, L. A. M. (2020). A saúde mental dos profissionais de saúde frente à pandemia do COVID-19: uma revisão integrativa. Revista Eletrônica Acervo Saúde, (46), e4128-e4128.

Schmidt, B., Crepaldi, M. A., Bolze, S. D. A., Neiva-Silva, L., \& Demenech, L. M. (2020). Saúde mental e intervenções psicológicas diante da pandemia do novo coronavírus (COVID-19). Estudos de Psicologia (Campinas), 37.

Silva, G. A., \& Otta, E. (2014). Revisão sistemática e meta-análise de estudos observacionais em Psicologia. Revista Costarricense de Psicología, 33(2), 137153.

Silva, F.,et al. Flaviane Cristine Troglio da; NETO, Modesto Leite. (2020). Psychiatric symptomatology associated with depression, anxiety, distress, and insomnia in health professionals working in patients affected by COVID19: a systematic review with meta-analysis. Progress InNeuroPsychopharmacology And Biological Psychiatry, 104, 110057-110064.

Silva, G. A., \& Otta, E. (2014). Revisão sistemática e meta-análise de estudos observacionais em Psicologia. Revista Costarricense de Psicología, 33(2), 137153. 
Research, Society and Development, v. 11, n. 2, e32411225706, 2022

(CC BY 4.0) | ISSN 2525-3409 | DOI: http://dx.doi.org/10.33448/rsd-v11i2.25706

Siqueira, A. B. R., dos Santos, M. A., \& Leonidas, C. (2020). Confluências das relações familiares e transtornos alimentares: revisão integrativa da literatura. Psicologia Clínica, 32(1), 123-149.

Sousa Júnior, B. S., de Mendonça, A. E. O., de Araújo, A. C., da Costa Santos, R., Neto, F. A. D., \& da Silva, R. A. R. (2020). Pandemia do coronavírus: estratégias amenizadoras do estresse ocupacional em trabalhadores da saúde. Enfermagem em Foco, 11(1. ESP).

Talevi, D., Socci, V., Carai, M., Carnaghi, G., Faleri, S., Trebbi, E., ... \& Pacitti, F. (2020). Mental health outcomes of the CoViD-19 pandemic. Rivista di psichiatria, 55(3), 137-144.

Xiao, C. (2020). A novel approach of consultation on 2019 novel coronavirus (COVID-19)-related psychological and mental problems: structured letter therapy. Psychiatry investigation, 17(2), 175.

Whittemore, R., \& Knafl, K. (2005). The integrative review: updated methodology. Journal of advanced nursing, 52(5), 546-553. 